\title{
Subdivision Schemes for Thin Plate Splines
}

\author{
Henrik Weimer Joe Warren \\ Department of Computer Science, Rice University \\ P.O. Box 1892, Houston, Texas 77005-1892, USA \\ $\{$ henrik, jwarren\}@cs.rice.edu
}

\begin{abstract}
Thin plate splines are a well known entity of geometric design. They are defined as the minimizer of a variational problem whose differential operators approximate a simple notion of bending energy. Therefore, thin plate splines approximate surfaces with minimal bending energy and they are widely considered as the standard "fair" surface model. Such surfaces are desired for many modeling and design applications.

Traditionally, the way to construct such surfaces is to solve the associated variational problem using finite elements or by using analytic solutions based on radial basis functions. This paper presents a novel approach for defining and computing thin plate splines using subdivision methods. We present two methods for the construction of thin plate splines based on subdivision: A globally supported subdivision scheme which exactly minimizes the energy functional as well as a family of strictly local subdivision schemes which only utilize a small, finite number of distinct subdivision rules and approximately solve the variational problem. A tradeoff between the accuracy of the approximation and the locality of the subdivision scheme is used to pick a particular member of this family of subdivision schemes.

Later, we show applications of these approximating subdivision schemes to scattered data interpolation and the design of fair surfaces. In particular we suggest an efficient methodology for finding control points for the local subdivision scheme that will lead to an interpolating limit surface and demonstrate how the schemes can be used for the effective and efficient design of fair surfaces.
\end{abstract}

Keywords: surface modeling, variational design, subdivision, thin plate splines, optimization, scattered data interpolation, fair surfaces.

\section{Introduction and Motivation}

The classical problem of geometric design is the definition and manipulation of curved shapes. For almost all modeling problems the represented shapes have to be visually appealing or mechanically stable. This in turn implies that certain conditions on the derivatives of the surface of the modeled object are satisfied. One very traditional approach to construct such surfaces is variational design where the surface model has to minimize a certain quadratic functional built from a set of differential operators. The quality of the surface is then measured in one scalar number by integrating this functional over the surface.

Thin plate splines are such a surface model where one wants the bending energy of the surface to be minimal. The minimality of the bending energy implies that the surface tangents vary as little as possible which explains the term variational design.

The first sections of this paper present a method to model thin plate splines on bounded rectilinear grids through a subdivision process. While the resulting subdivision scheme exactly reproduces this particular family of splines it is globally supported. The second half of this paper focuses on the derivation of a family of local subdivision schemes which behave almost like thin plate splines. These schemes use only a small number of distinct subdivision rules that can be precomputed entirely. The resulting subdivision surfaces can be manipulated extremely efficiently. Locality will be the parameter for choosing a particular member of this family of subdivision schemes and the exactness of the approximation of thin plate splines will depend on the support. 


\subsection{Thin Plate Splines}

Thin plate splines were introduced to geometric design by Duchon ${ }^{6}$ in 1976 as the minimizer of the functional

$$
\mathcal{E}(f)=\int_{\mathbb{R}^{2}}\left(f_{u u}^{2}+2 f_{u v}^{2}+f_{v v}^{2}\right) d u d v
$$

with respect to some additional interpolation constraints. Thus, a thin plate spline can be defined as the function $F$ such that

$$
\begin{array}{ll}
\text { - } & \mathcal{E}(F) \text { is minimal } \\
\text { - } & F\left(T_{0}\right)=c_{0}
\end{array}
$$

for a given set of values $c_{0}$ at a given set of knots $T_{0} . \mathcal{E}(f)$ approximately measures the bending energy of a thin, infinitely extending elastic plate which is fixed in the interpolation points $c_{0}$.

The Euler-Lagrange equation implies that solutions to the variational problem (2) will satisfy the biharmonic equation

$$
F_{u u u u}+2 F_{u u v v}+F_{v v v v}=0
$$

everywhere in the domain except at the location of original knots $T_{0}{ }^{10}$.

Thin plate splines have global support, i.e. at any point in the resulting surface all of the data points $c_{0}$ have a nonzero influence. Furthermore they have linear precision which states that if the given set of interpolation points $c_{0}$ lies in a plane then the resulting spline will reproduce this plane. Moreover they are invariant under translation, rotation and uniform scaling.

Various techniques for solving this particular variational problem have been used. Traditionally, finite element methods ${ }^{1,19}$ can be applied to approximate the solution in an iterative solution process. Alternatively logarithmic radial basis functions can be used to express the solution analytically ${ }^{18}$.

\subsection{Subdivision}

Subdivision is a way of representing curved shapes as the limit of iterated linear transformations which map coarse sets of control points to finer ones. A subdivision scheme is described by a sequence of subdivision matrices $S_{k}$ which map control points $p_{k}$ of level $k$ to control points $p_{k+1}$ at level $k+1$ by

$$
p_{k+1}=S_{k} p_{k} .
$$

The actual subdivision object (curve, surface, volume and so on) is now defined as the limit of this process,

$$
\lim _{k \rightarrow \infty} p_{k}
$$

A subdivision scheme is called local if the computation of a new control point at level $k+1$ only involves old control points of level $k$ which lie in the topological neighborhood of the new point.
A very classical example of such a local subdivision process is the Lane-Riesenfeld algorithm for repeated knot insertion for B-splines ${ }^{15}$ which is shown in figure 1 for a uniform cubic spline. Depicted are the control points for level 0 , 1 and 2 from top to bottom. The subdivision matrix $S_{0}$ which maps the five control points of level 0 into nine control points of level 1 has the form

$$
S_{0}=\frac{1}{8}\left(\begin{array}{lllll}
8 & 0 & 0 & 0 & 0 \\
4 & 4 & 0 & 0 & 0 \\
1 & 6 & 1 & 0 & 0 \\
0 & 4 & 4 & 0 & 0 \\
0 & 1 & 6 & 1 & 0 \\
0 & 0 & 4 & 4 & 0 \\
0 & 0 & 1 & 6 & 1 \\
0 & 0 & 0 & 4 & 4 \\
0 & 0 & 0 & 0 & 8
\end{array}\right)
$$

and the subdivision matrices $S_{k}$ for $k>0$ have a similar structure. In particular, most of the columns of any $S_{k}$ are shifts of the center column of $S_{0}$. Special columns appear only at both ends of the knot vector. For uniform univariate cubic B-splines the subdivision matrices contain only three different kinds of columns and three different kinds of rows which are entirely predetermined.

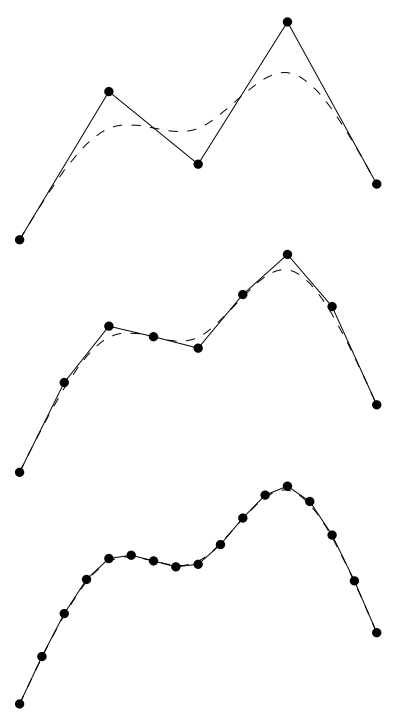

Figure 1: Approximating subdivision for uniform cubic Bsplines: The original control polygon is shown on the top. The middle and bottom depict the control polygon after one and two rounds of subdivision. The final spline is depicted as a dashed curve.

Note that in figure 1 the limit curve does not interpolate the original control points $p_{0}$ and that the sequence of control polygons converges to the limit curve very quickly. Subdivision schemes with the property that the limit object does not interpolate the control points are called approximating. 
The benefits of subdivision lie in the simplicity and generality of the approach. Implementation of a subdivision scheme is very simple because only polyhedral models are needed. Furthermore the subdivision scheme implicitly defines several level of detail representations of the limit object, depending on the number of steps the subdivision process has been applied. Subdivision schemes are a very powerful way of representing a function because no explicit basis for the solution space is needed. In particular it is not necessary to know whether the solution space is polynomial, rational, logarithmic, hyperbolic and so on.

Highly desirable are subdivision schemes that are local and for which the actual subdivision rules can be constructed easily. In this case the resulting surfaces can be computed and manipulated extremely efficiently and the relation between the original control points and the limit surface is fairly intuitive. The most successful subdivision schemes in practical use today are local and rely on precomputed subdivision rules, e.g. 15, 7, 16, 9, 23, 24 .

This paper will make use of the methodology introduced by Warren in 22 and we will show how it can be used to construct schemes for one of the most interesting variational design problems - thin plate splines. Using this approach, the solution to this particular variational problem can be recast in the framework of subdivision and all the nice properties and algorithms for subdivision are applicable.

Unfortunately for the exact subdivision scheme the actual subdivision rules are neither local nor cheap to compute. In particular, the subdivision rules have to be recomputed at every level in the scheme.

Many applications in geometric design do not require exact minimization of the thin plate spline energy functional (1). One rather desires smooth surfaces which are fair, wellbehaved and can be computed efficiently. Minimizing the bending energy functional only guides the surface in that direction. Therefore the main point of this paper will be the derivation of a family of subdivision schemes for surfaces which mimic the behavior of thin plate splines. These subdivision schemes are local and rely only on a small number of precomputed subdivision rules. They produce smooth limit functions which almost solve the original variational problem and can be evaluated extremely efficiently.

The distinguishing parameter for the members in this family of subdivision schemes will be the support of the subdivision matrix. The larger the support the more exact the approximation of the original thin plate spline will be, but also the less efficient can the surfaces be manipulated. Thus choosing a particular member of this family involves a tradeoff between accuracy and efficiency of the solution.

\subsection{Related Work}

Variational design, thin plate splines and subdivision have all been studied very intensively over the past decades. We see our work mainly influenced by the following sources:

As mentioned earlier, Duchon 6 introduced thin plate splines to geometric design. Meinguet ${ }^{18}$ suggested their use for scattered data interpolation. Finally Franke ${ }^{8}$ extended thin plate splines by adding tension parameters to the energy functional. More conventionally and in the general case variational problems are solved by finite element approaches 1,19 .

Modeling through subdivision became a hot topic in geometric design after the two landmark papers by Catmull and Clark 2 and Doo and Sabin 5 in the late 1970s. The schemes introduced by Dyn, Levin and Gregory ${ }^{7}$, its subsequent improvement for irregular meshes by Zorin et al. ${ }^{23,24}$ and in particular Loop's subdivision scheme 16,9 found many practical applications. A comprehensive textbook on subdivision will become available soon ${ }^{21}$.

Smooth discrete interpolation based on the minimization of a roughness criterion was presented by Mallet ${ }^{17}$. More recently the idea of variational subdivision has been discussed by Kobbelt and Schröder for interpolating curves 12,14 as well as for the fairing of interpolating triangular meshes using the thin plate spline energy functional 13. A general methodology which can be used to solve elliptic variational problems using approximating subdivision schemes has been presented in 22 .

The work presented here is distinguished from previous work on variational subdivision for surfaces by the use of an approximating subdivision basis. The use of approximating basis functions leads, just as for B-splines in the one dimensional case, to basis functions which are highly local. In the end this enables us to find truly local subdivision schemes which almost solve the variational problem while utilizing only a small number of precomputed rules. Thus, these local schemes are much more efficient in practical use

\section{Variational Subdivision}

The idea of variational subdivision is to represent the solution to a variational problem that is described in terms of its differential operators through a subdivision process. Warren 22 presented a paradigm for deriving approximating subdivision schemes which exactly solve variational problems. This framework will serve as the basis for our derivations in this paper and it will be augmented by a methodology for deriving purely local subdivision schemes which almost satisfy the variational description of thin plate splines. These local subdivision schemes utilize only a small number of different subdivision rules which can be entirely precomputed.

The goal is to represent the solution of the variational thin plate problem (1) as the limit of a subdivision process. 
Later, the behavior of this subdivision scheme for thin plate splines is approximated by a small set of local subdivision rules which can be precomputed once. The remainder of this section will briefly outline the method presented in ${ }^{22}$. For a more detailed and theoretically justified exposition see the original reference.

\subsection{Energy and Subdivision}

As pointed out earlier in the introduction thin plate splines are defined as the minimizers of the energy functional (1)

$$
\mathcal{E}(f)=\int_{\mathbb{R}^{2}}\left(f_{u u}^{2}+2 f_{u v}^{2}+f_{v v}^{2}\right) d u d v .
$$

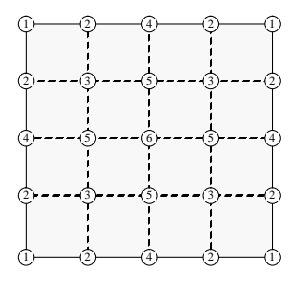

difference mask location

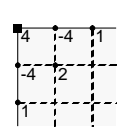

(1)

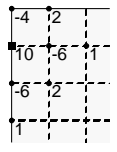

(2)

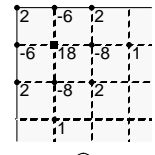

(3) corner masks
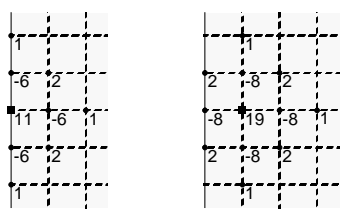

(4)

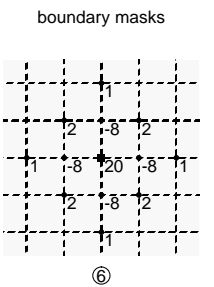

interior mask

Figure 2: Structure of the energy matrix $E$ for thin plate splines. The top row depicts a rectilinear grid with the knots numbered. The remaining rows depict the rules used to compute a discrete energy value for the different kinds of knots in the grid.

Solving this problem in the discrete framework of subdivision requires recasting of this integral energy measure $\mathcal{E}$ to a discrete setting. Given a set of control points $p_{k}$ for level $k$ of the subdivision process, a continuous version of this subdivision representation can be defined by choosing a set of basis functions $B_{k}$ and writing the level $k$ solution as

$$
F_{k}(u, v)=B_{k}(u, v) p_{k} .
$$

Then the energy of the control points $p_{k}$ can be defined as the energy of $F_{k}$ with respect to the original energy functional (1), i.e. as $\mathcal{E}\left(F_{k}\right)$, which yields the quadratic form

$$
\mathcal{E}\left(F_{k}\right)=p_{k}^{T} E_{k} p_{k} \text {. }
$$

$E_{k}$ is a square matrix, called the energy matrix. Given an arbitrary set of control points $p_{k}$ for level $k$ the energy of the control grid can now be assessed using this energy matrix. The entries in $E_{k}$ are inner products of appropriate derivatives of finite element basis functions centered over the knots of the level $k$ grid.

This method for computing the energy of the thin plate spline $F_{k}$ with control points $p_{k}$ is theoretically sound and general and is applicable in the case of irregular grids as well. Because its implementation is fairly involved we will be using the well known difference masks for the biharmonic equation (4) instead which can be found in any text book on finite differences, e.g. ${ }^{19}$. These difference masks are depicted in figure 2 . The reader familiar with finite element methods may recognize these energy masks as the difference schemes for the biharmonic equation.

\subsection{Variational Subdivision for Thin Plate Splines}

The idea of approximating variational subdivision as presented in 22 is to compute the control points for the finer grid in such a way that the energy of the control grid is preserved at old control points and energy zero is achieved at all new control points.

To formalize this constraint we make use of an upsampling matrix $U_{k}$ which carries coefficients associated with control points at level $k$ over to level $k+1$ by inserting coefficient zero for all control points which are new at level $k+1$. Figure 3 illustrates the action of the upsampling matrix: Depicted are three successive levels of subdivision of a $3 \times 3$ grid. The energy value associated with the original, leftmost control points are painted as black dots. The energy values inserted through upsampling are shown as light gray dots. Note that the subdivided grids will maintain the old energy values (black dots) at the location of original, level 0 control points, while a discrete energy of zero (gray dots) is inserted for all new knots. Intuitively the limit of this process will produce coefficients that satisfy the biharmonic equation (4) everywhere except at the original knots.

This idea leads to a constraint on the subdivision matrix for level $k$ which can be expressed as

$$
E_{k+1} S_{k}=U_{k} E_{k}
$$

where $E_{k}$ denotes the energy matrix defined in (9), $S_{k}$ is the subdivision matrix for level $k$ and $U_{k}$ represents upsampling 

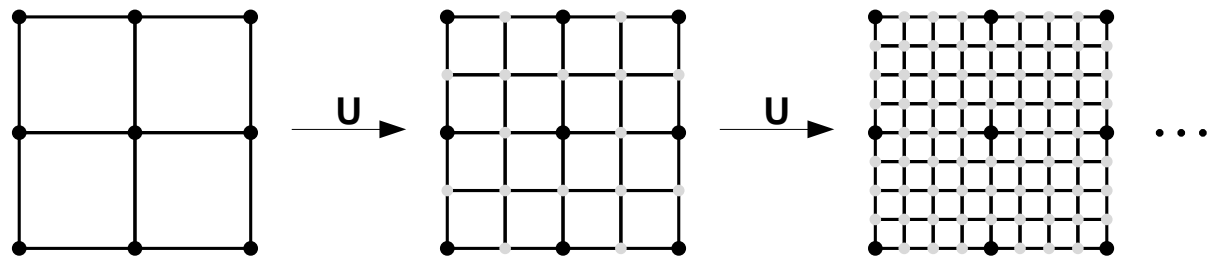

Figure 3: The action of the upsampling matrix $U$. Zero energy values are depicted by light gray dots, non-zero energy is shown as black dots. In the limit this process will produce discrete energy zero everywhere except at the original knots.

from level $k$ to level $k+1$ by inserting zeros inbetween old coefficients.

Equation (10) states that subdividing the control points and computing the energy of the resulting finer grid (left hand side) should be the same as computing the energy at the coarse level and upsampling these values to the finer grid by inserting zeros inbetween (right hand side).

If the subdivision matrices $S_{k}$ satisfy equation (10) then the limit surface of the subdivision process is the minimizer of the original variational problem. For a complete formal proof see 22 .

As the energy matrices $E_{k}$ are rank deficient, the subdivision matrices $S_{k}$ are not well defined by (10) alone. Additional constraints have to be added to choose one particular subdivision basis for the solution space. For example, one reasonable property the subdivision scheme should have is the preservation of moments which states that the inner products between lower degree polynomials and the finite element basis function used for the construction of $E$ should remain constant under subdivision. If $M$ is a matrix representing the expression $M=\int u^{i} v^{j} B_{k}(u, v) d u d u$ for some $i, j \geq 0$ then this constraint can be expressed as

$$
M_{k+1} S_{k}=M_{k} .
$$

This additional constraint on $S_{k}$ will lead to a unique and well-behaved subdivision scheme. For thin plate splines the constant and linear moments $(i, j)=(0,0),(i, j)=(1,0)$, $(i, j)=(0,1)$ have to be considered

Solving equation (10) yields subdivision matrices $S_{k}$ which will produce the exact minimizer of the variational problem as their limit surface. Figure 4 depicts three different stencils of a particular $S$. Shown are rows from the interior of the grid rounded to five digits. The exact stencils are globally supported and have been truncated to a reasonable size. Shown are the rules to compute a new coefficient for a vertex, an edge and a face in the interior of the grid. The location of the new control point is depicted by a box.

Inspection of the resulting subdivision matrices suggests the following statements:

- The subdivision rules do not depend significantly on the location in the grid. Rules closer to the boundary are different from the ones in the interior, but the subdivision

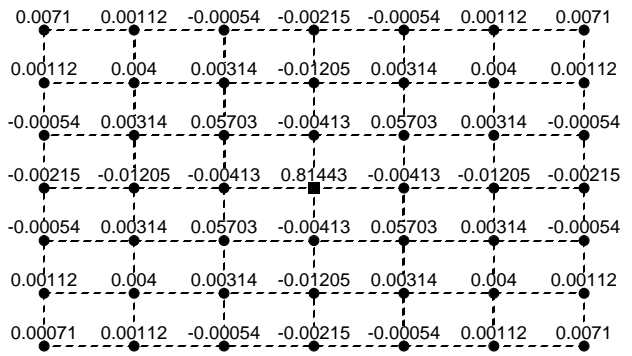

vertex mask
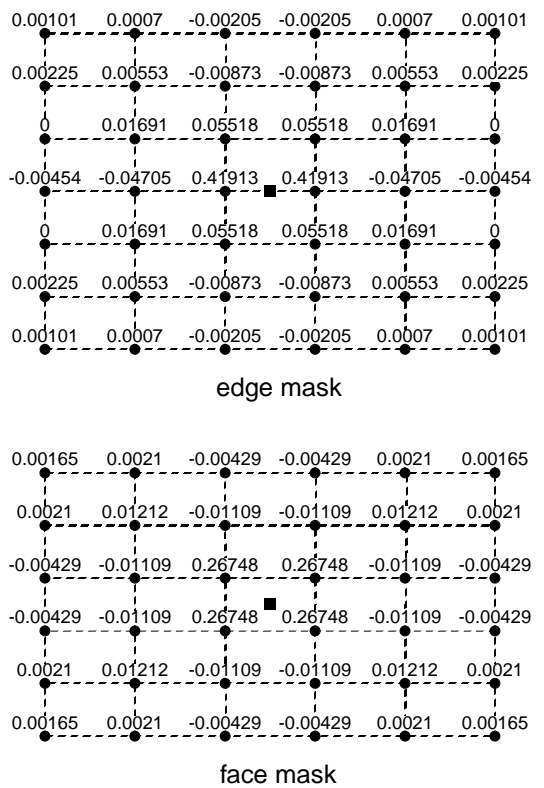

Figure 4: Exact subdivision masks for thin plate splines rounded to five digits and truncated.

rules for neighboring vertices in the interior of the grid are almost identical, so are those for two neighboring edges or faces.

- The rules do not depend significantly on the level $k$ of the subdivision. Rules for vertices, edges and faces at level $k$ are almost identical to the respective rules for level $j \neq k$. 


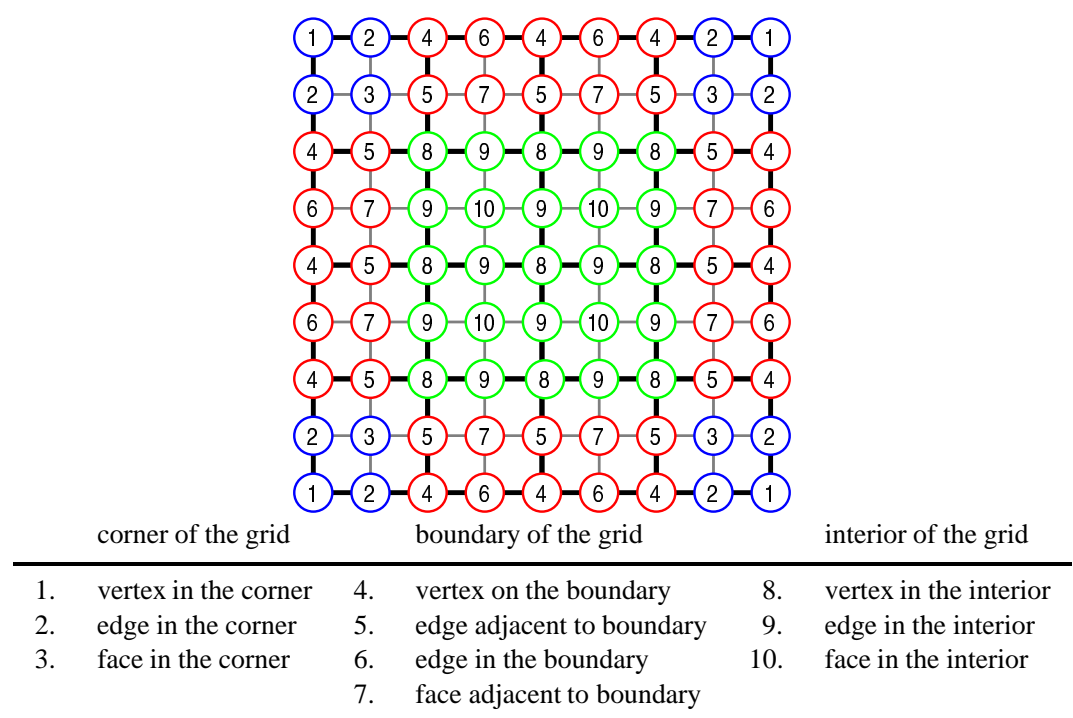

Figure 5: Structure of the subdivision matrix shown for a $5 \times 5$ grid. Grid lines of the coarse grid are solid, grid lines of the fine grid are gray.

- The rules are almost local, i.e. for a new control coefficient the old coefficients in its neighborhood have a very large influence while control coefficients further away have very small weights.

The subdivision scheme presented in this section is not local but it produces the exact thin plate spline as the limit surface. The subdivision matrix has to be recomputed for every level in the subdivision process and it is globally supported.

\section{A Local Subdivision Scheme for Thin Plate Splines}

The observations that the subdivision rules have fast decay, look very similar at different levels, and are mostly independent of the actual location in the mesh suggest that the scheme could be approximated to a high degree of accuracy by a local subdivision scheme which utilizes only a small number of distinct subdivision rules. This section focuses on the definition of a family of such subdivision schemes. An actual member of this family is picked by specifying the support of the subdivision basis functions and we demonstrate here how to compute the scheme with the same support as bicubic B-splines, i.e. $5 \times 5$ in a rectilinear grid.

These local subdivision schemes no longer exactly solve equation (10). Thus, they no longer produce the exact minimizer of the variational problem (1). For many geometric design applications this exact energy minimization is not critical. One is rather interested in having an efficient algorithm for constructing fair and smooth surfaces. We consequently believe that there are many real world applications which can make use of such subdivision schemes which almost reproduce thin plate splines.
To allow for stability and local support in the subdivision process, the exact equality constraint in (10) has to be relaxed. The original equation (10) reduces to

$$
E_{k+1} \hat{S}_{k} \approx U_{k} E_{k}
$$

where $\hat{S}_{k}$ denotes the local subdivision matrix. One very reasonable method of solving for the entries of $\hat{S}_{k}$ is to set up the system (12) symbolically and then to minimize the $L_{\infty}$-norm of the expression

$$
\left\|E_{k+1} \hat{S}_{k}-U_{k} E_{k}\right\|_{\infty}
$$

which will lead to a linear programming problem. Minimization of the $L_{\infty}$-norm of this problem is preferred over e.g. the $L_{2}$-norm because convergence of a subdivision scheme is determined in the $L_{\infty}$-norm.

Equation (13) defines a whole family of local subdivision schemes which approximate thin plate splines. The actual subdivision scheme is determined by the particular support and structure of the subdivision matrix $\hat{S}_{k}$ and the level $k$ of subdivision. In the following section it will become clear why the actual solution is invariant under the level $k$. The locality and structure of the subdivision matrix remain as the only parameters for picking an actual member of this family of subdivision schemes. We will show how to find the scheme with support equivalent to bicubic B-splines on a rectilinear grid.

\subsection{Building the Local Subdivision Matrix from a Small Set of Rules}

Given a particular grid of level $k$ a local subdivision matrix $\hat{S}_{k}$ can be constructed which uses only a small number 
of distinct subdivision rules. The remainder of this section will focus on the process of building this symbolic representation of the subdivision matrix and solving the resulting linear program.

The rows of the $\hat{S}_{k}$ are picked depending on the location of the new control point in the grid. For the subdivision scheme with the support of bicubic B-splines we suggest using different rules in the subdivision scheme as shown in figure 5 . Each entry in the table in figure 5 corresponds to a different type of row in the subdivision matrix. All rows of a given type are equivalent. Symmetries and simple shifts can be used to compute the actual stencil for a particular control point.

The second point of concern is the actual support of the rows of the symbolic subdivision matrix. We elect to classify the locality of the subdivision schemes by the support of the columns of the subdivision matrix. A column of the subdivision matrix describes how an old control coefficient is split over new coefficients. In general, a column support of $(2 n+1) \times(2 n+1)$ can be chosen for any $n \geq 0$. Only odd supports make sense because the rules should be symmetric and the columns are centered over the old coefficient. A column support of $(2 n+1) \times(2 n+1)$ implies that the subdivision stencil to compute a new face will have support $2(n-1) \times 2(n-1)$. The stencil for a vertex has support $\left(2\left\lfloor\frac{n}{2}\right\rfloor+1\right) \times\left(2\left\lfloor\frac{n}{2}\right\rfloor+1\right)$ and a control coefficient for an edge has support $\left(2\left\lfloor\frac{n}{2}\right\rfloor+1\right) \times 2(n-1)$. Along the grid boundaries the columns of the subdivision matrix certainly only contribute to the interior of the grid.

If the subdivision basis functions should have the same support as bicubic B-splines then column $i$ of $\hat{S}$ will have a $5 \times 5$ support centered over control point $i$. This implies that the rows of $\hat{S}$ have the stencils shown in figure 6 , e.g. a new control point for an interior face is supported over the corner vertices of the face, a control point centered over a interior vertex of the coarse grid is supported over the coefficients centered over the knot itself and the eight neighboring knots and so on. The actual structure of the particular subdivision rules is also depicted in figure 6 . Two control points will receive the same weight due to symmetry when the corresponding knots have the same icons in the figure.

Given the symbolic representation of $\hat{S}$ a linear program for (13) can be constructed by choosing a sufficiently large $k$, constructing $\hat{S}_{k}$ and then computing the norm in (13).

Observe that the $L_{\infty}$-norm of a matrix neither depends on the order of terms in a row of the matrix nor on the particular order of the rows. Therefore, the terms in the rows of matrix (13) as well as the rows themselves can be sorted into a canonical lexicographic order without changing the $L_{\infty}$-norm of the expression. Using simple set operations redundancies can be eliminated by discarding duplicate terms and rows. The norm of the matrix expression (13) is invariant under this reduction.

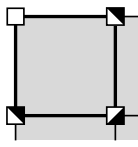

(1)

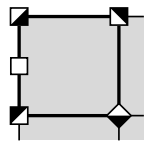

(2)

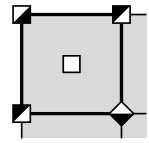

(3)

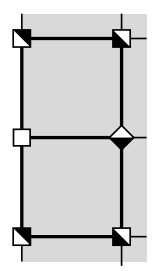

(4)

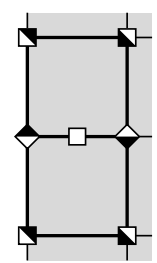

(5)

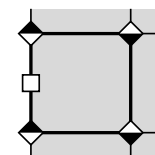

(6)

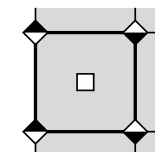

(7)

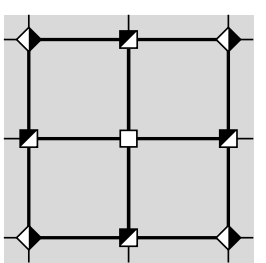

(8)

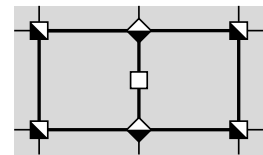

(9)

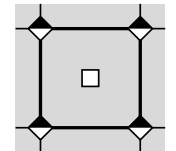

(10)
Figure 6: The support and structure of the different kinds of rows in the subdivision matrix: The interior of the grid is shown as a gray shaded region and the location of the new control point is depicted by an empty box. Indices refer to figure 5. Inside one rule all coefficients with the same icon will have the same value, e.g. in rule 1 the top right and bottom left coefficients are forced to be equal while the two remaining entries are unconstrained.

Closer observation reveals that in fact expression (13) is independent of the level $k$ of the subdivision. Because $\hat{S}$ uses only a fixed, small number of distinct rules, two subdivision matrices $\hat{S}_{k}$ and $\hat{S}_{l}$ have similar rows which are shifts of each other. Furthermore the energy matrices $E_{k}$ and $E_{l}$ have similar rows. Multiplication of $E$ and $S$ thus also leads only to a finite number of different, similar rows. The redundancy elimination described above determines the unique, minimal set of distinct rows in this process independent of $k$. Once a certain threshold for $k$ has been passed all the different kinds of non-zero combinations of rows of $S$ and $E$ show up in (13) and the redundancy elimination reduces these rows to a minimal set of expressions which entirely determine the $L_{\infty}$-norm.

For the subdivision scheme with the support of bicubic B-splines and the structure previously described in figures 5 and 6 the system reaches its maximal size after 5 rounds of subdivision. The redundancy elimination produces in this case a set of 21 distinct rows in 83 distinct terms for expression (13).

A small linear program for this reduced set of constraints can be constructed. After adding three additional linear con- 
straints to assert constant and linear precision of the subdivision scheme, the system can be solved once using any reasonable linear programming package, e.g. CPLEX ${ }^{4}$. The result of this linear optimization are coefficients for the different kinds of subdivision rules in $\hat{S}$. These can be used to construct subdivision matrices for arbitrary size rectilinear grids at arbitrary levels of subdivision. The different rules resulting from solving this process for subdivision matrices with the $5 \times 5$ column support of bicubic B-splines are shown in figure 7 .

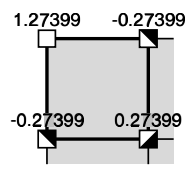

(1)

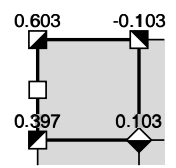

(2)

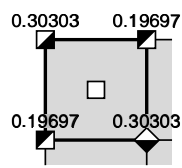

(3)

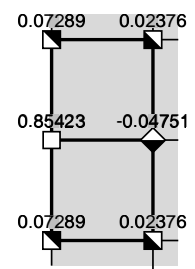

(4)

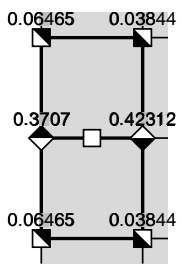

(5)

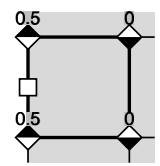

(6)

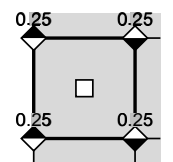

(7)

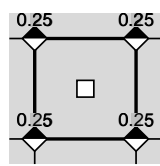

(10)

Figure 7: The rules of the local subdivision scheme for the approximation of thin plate splines. Indices refer to figure 5 .

The subdivision process does not produce the exact minimizer to the variational problem but it is highly local and can be entirely precomputed - thus it can be applied extremely efficiently.

\section{Properties of the Subdivision Schemes}

The exact solution to the thin plate spline variational problem has $C^{\infty}$-continuity everywhere except at the location of the original knots - there it is just $C^{1}$. The global subdivision scheme presented in section 2.2 solves equation (10). Thus, it exactly reproduces the minimizer of the variational problem (2) ${ }^{22}$. Therefore, the exact subdivision scheme produces limit surfaces which are $C^{\infty}$-continuous everywhere except at the location of original knots - there they are just $C^{1}$.

Analyzing the smoothness of the local schemes proves slightly more involved. The eigen-structure of the schemes

can be analyzed. The eigen-spectrum of the particular local scheme presented in the previous section contains leading eigen-values $1,0.5,0.5,0.37, \cdots$ with all the remaining values having modulus smaller than 0.37 . Thus the eigenspectrum is consistent with the subdivision scheme being $C^{1}$-continuous ${ }^{3,20}$. Furthermore the scheme appears visually smooth in all our examples.

Note that any degree of continuity can be enforced by adding appropriate difference constraints for the subdivision scheme to the linear program and at the same time increasing the column-support of $\hat{S}$.

\section{Applications}

This section presents applications of the approximating subdivision process. We will show how the scheme can be used to interpolate a given set of values and apply it to define fair surfaces. Note that to apply the subdivision scheme we do not have to re-run the optimization process in (13). Instead, the coefficients in figure 7 are used.

\subsection{Control Points for Interpolating Subdivision Surfaces}

The presented subdivision schemes are approximating. This means that the subdivision surface does not interpolate the vertices of the original control net. Like in the case of Bspline curves, the sequence of control nets describes closer and closer approximations of the limit surface. Hence, in order to interpolate a particular set of points, adequate initial control coefficients for the subdivision have to be determined first.

Formally this problem can be described as follows: Given a set of interpolation conditions $c_{0}$ centered over the knots of the original control grid, find a set of initial control points $p_{0}$ centered over the same knots such that

$$
I_{0} p_{0}=c_{0} .
$$

Here $I_{0}$ denotes the interpolation matrix for level 0 . The interpolation matrix $I_{k}$ contains the values of the basis functions which are induced by the subdivision scheme sampled at the knots of level $k$. The $(i, j)$-th entry of $I_{0}$ contains the value of the basis function centered over knot $i$ sampled at knot $j$.

The interpolation matrix $I_{k}$ is characterized by the equation

$$
U_{k}^{T} I_{k+1} S_{k}=I_{k}
$$

As mentioned earlier $I_{k}$ contains the values of the basis functions of level $k$ sampled at the knots. Equation (15) states that computing the values of the basis functions at level $k$ (right hand side) is the same as subdividing the basis functions, then computing their values at the finer grid and then downsampling back to the coarse grid using the transpose of the upsampling matrix (left hand side). 
The subdivision scheme presented in section 3 is local and uses only a small number of precomputed rules. Thus, the rows of the interpolation matrices $I_{k}$ are also drawn from a small set of common rows. In fact, the support of the interpolation matrix $I_{k}$ is the same as the support of $U_{k}^{T} S_{k}$. A symbolic representations of $I_{k}$ can be constructed easily using the representation for $S_{k}$. Together with an additional constraint that the rows of $I_{k}$ have to sum to one the interpolation matrices are completely determined by equation (15). A linear program for $\left\|U_{k}^{T} I_{k+1} S_{k}-I_{k}\right\|_{\infty}=0$ can be used to solve for the entries in $I$.

Given a set of interpolation constraints $c_{0}$ centered over the knots of level 0 a suitable set of control points $p_{0}$ can then be determined very efficiently using the inverse of the interpolation matrix $I_{0}$ :

$$
p_{0}=I_{0}^{-1} c_{0} .
$$

The following section extends this paradigm to the case where the set of interpolation constraints $c_{0}$ does not cover the full set of knots of level 0 .

\subsection{Scattered Data Interpolation}

For this example the scattered data problem is, given a set of functional samples $\left\{\left(u_{i}, v_{i}, w_{i}\right) \mid i=1, \cdots, n\right\}$ find a surface which interpolates these points.

Given such a set of samples an interpolating subdivision surface based on the local subdivision scheme from section 3 can be constructed by picking a sufficiently dense initial grid of knots and then warping the samples $\left(u_{i}, v_{i}, w_{i}\right)$ to the closest knot with respect to $u$ and $v$ as shown in figure 8 . The value $w_{i}$ is used as the desired interpolation value associated with the knot.
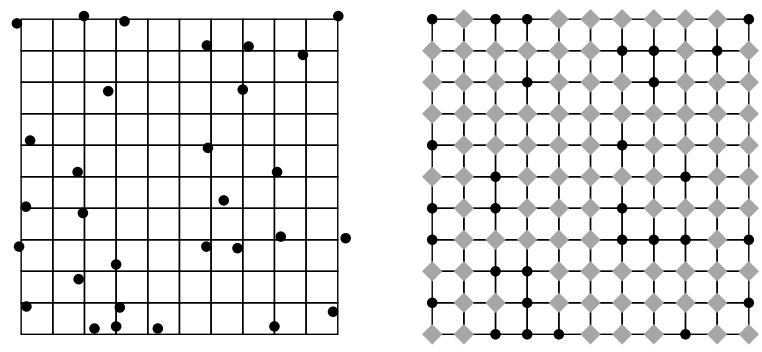

Figure 8: The samples (solid dots) are warped to knots of a grid based on $u$ and $v$. This leads only to interpolation conditions for a subset of the knots. The remaining control points, depicted by gray diamonds, can be determined by constraining the discrete energy at the knot to be zero.

As the set of samples usually does not cover all of the knots, only a subset of them have associated interpolation conditions. Thus, it is not possible to find the complete control net by simply applying the inverse of the interpolation matrix as described in the previous section. Instead, the control points have to be computed such that interpolation is achieved at those knots which have an associated interpolation constraint and energy zero is achieved at all the remaining knots. This construction will produce a control coefficient associated with every knot and the subdivision scheme can be applied. We plan to publish a paper focusing on the details of this method at a later time.

An example is shown in figure 9. The original surface was given by

$$
\begin{aligned}
f(u, v)= & -0.4 e^{-\left((u-1)^{2}+(v+1)^{2}\right)} \\
& +0.6 e^{-\left((u+1.2)^{2}+(v+1.2)^{2}\right)} \\
& +e^{-\left((u-1)^{2}+(v-1)^{2}\right)} .
\end{aligned}
$$

\subsection{Design of Fair Surfaces}

A very common problem in geometric design is the modeling of fair surfaces. In this framework a fair and smooth has to be constructed which matches up with existing curves along patch boundaries. This section will demonstrate how this problem can be solved efficiently using our local subdivision scheme.

The naive method for applying a functional subdivision scheme to a three-dimensional problem is to apply the rules separately to the three parameter functions. The resulting surface is smooth but the variational properties of the object can not be guaranteed. A more reasonable solution is to locally reparametrize the mesh by projecting the local neighborhood into an estimate for the tangent plane and applying the scheme in the reparametrized space.

An example application for the design of fair surfaces in $3 \mathrm{D}$ is shown in figure 10. A polygonal model of the space shuttle has been modified to replace the part representing the nose by a dense mesh generated through the subdivision scheme described in section 3 . The fair surface shown here interpolates the vertices that were present in the original model. The control points for the subdivision were determined using the inverse of the interpolation matrix.

\section{Conclusion and Future Work}

We presented two subdivision schemes for thin plate splines on a rectilinear grid. The first scheme reproduces the exact solution to the variational problem. It has global support and the subdivision matrix has to be recomputed at every level of the subdivision process. The second scheme is local and uses only a small number of rules which can be precomputed, but it only approximates thin plate splines. The quality of the approximation depends on the support of the subdivision mask. Arbitrarily small residuals can be achieved by increasing the support of the basis functions.

In the future, we plan to extend this approach to handle irregular grids of arbitrary topological type. We first plan to 
work out a formulation of the exact subdivision scheme for thin plate splines in this framework by generalizing the energy matrix to irregular grids. The ultimate goal will be to find again a simple set of subdivision rules that can be used to approximate the exact solution to a high degree of accuracy. Because the local geometry of the grid can be arbitrary in the case of irregular grids it may be necessary to parameterize the subdivision rules over the local geometry.

Finally, we plan to generalize the method for constructing interpolation matrices to local, stationary subdivision schemes, such as Loop's scheme ${ }^{16}$.

\section{Acknowledgments}

This work has been supported in part under National Science Foundation grant CCR-9500572, Texas Advanced Technology Program grant 003604-010 and by Western Geophysical.

\section{References}

1. S. Brenner and L. Scott, The mathematical theory of finite element methods, Springer-Verlag (1994).

2. E. Catmull and J. Clark, Recursively Generated BSpline Surfaces on Arbitrary Topological Meshes, Computer Aided Design 10, 6, pp. 350-355 (1978).

3. A. Cavaretta, W. Dahmen and C. Micchelli, Stationary Subdivision, Memoirs of the AMS, 453 (1991).

4. CPLEX Optimization, Inc, Using the CPLEX Callable Library (1995).

5. D. Doo and M. Sabin, Analysis of the Behavior of Recursive Division Surfaces near extraordinary Points, Computer Aided Geometric Design, 10, 6, pp. 356-360 (1978).

6. J. Duchon, Splines minimizing rotation invariant seminorms in Sobolev spaces, in W. Schempp and K. Zeller (eds.): Constructive Theory of Functions of several variables : proceedings of a conference held at Oberwolfach, April 25-May 1, 1976, Lecture Notes in Mathematics, 571, pp. 85-100, Springer-Verlag (1977).

7. N. Dyn, D. Levin and J. A. Gregory, A Butterfly Subdivision Scheme for Surface Interpolation with Tension Control, Transactions on Graphics, 9, 2, pp. 160-169 (1990).

8. R. Franke, Thin Plate Splines with Tension, in R. E. Barnhill and W. Böhm (eds.), Surfaces in CADG 1984, North-Holland, pp. 87-95 (1985),

9. H. Hoppe, T. DeRose, T. Duchamp, M. Halstead, H. Jin, J. McDonald, J. Schweitzer and W. Stuetzle, Piecewise Smooth Surface Reconstruction, Computer Graphics Proceedings, Annual Conference Series, pp. 295 300 (1994).
10. J. Hoschek and D. Lasser, Grundlagen der Geometrischen Datenverarbeitung, 2nd edition, B. G. Teubner (1992).

11. L. Kobbelt, A variational approach to subdivision, Computer Aided Geometric Design, 13, pp. 743-761 (1992).

12. L. Kobbelt, Interpolatory Subdivision on Open Quadrilateral Nets with Arbitrary Topology, Proceedings of Eurographics 1996, Computer Graphics Forum, pp. 409-420 (1996)

13. L. Kobbelt, Discrete Fairing, Proceedings of the Seventh IMA Conference on Mathematics of Surfaces (1996).

14. L. Kobbelt and P. Schröder, Constructing Variationally Optimal Curves through Subdivision, California Institute of Technology, Department of Computer Science, Technical Report CS-TR-97-05 (1997).

15. J. Lane and R. Riesenfeld, A Theoretical Development for the Computer Generation and Display of Piecewise Polynomial Surfaces, IEEE Transactions on Pattern Analysis and Machine Intelligence, PAMI-2, 1, pp. 35-46 (1980).

16. C. Loop, Smooth subdivision surfaces based on triangles, Master's thesis, Department of Mathematics, University of Utah (1987).

17. J.-L. Mallet, Discrete smooth interpolation in geometric modeling, Computer Aided Design, 24, 4, pp. 178191 (1992).

18. J. Meinguet, Multivariate Interpolation at Arbitrary Points Made Simple, Journal of Applied Mathematics and Physics, 30, pp. 292-304 (1979).

19. J. T. Oden and J. N. Reddy, An Introduction to the Mathematical Theory of Finite Elements, John Wiley \& Sons (1976).

20. U. Reif, A unified approach to subdivision algorithms near extraordinary vertices, Computer Aided Geometric Design, 12, pp. 153-174 (1995).

21. J. Warren, Subdivision methods for geometric design, to appear, Morgan Kaufman.

22. J. Warren, Subdivision schemes for variational problems, to appear in Computer Aided Geometric Design.

23. D. Zorin, P. Schröder and W. Sweldens, Interpolating Subdivision for Meshes with Arbitrary Topology, Computer Graphics Proceedings, Annual Conference Series, pp. 189-192 (1996)

24. D. Zorin, P. Schröder and W. Sweldens, Interactive multiresolution mesh editing, Computer Graphics Proceedings, Annual Conference Series, pp. 295-268 (1997). 

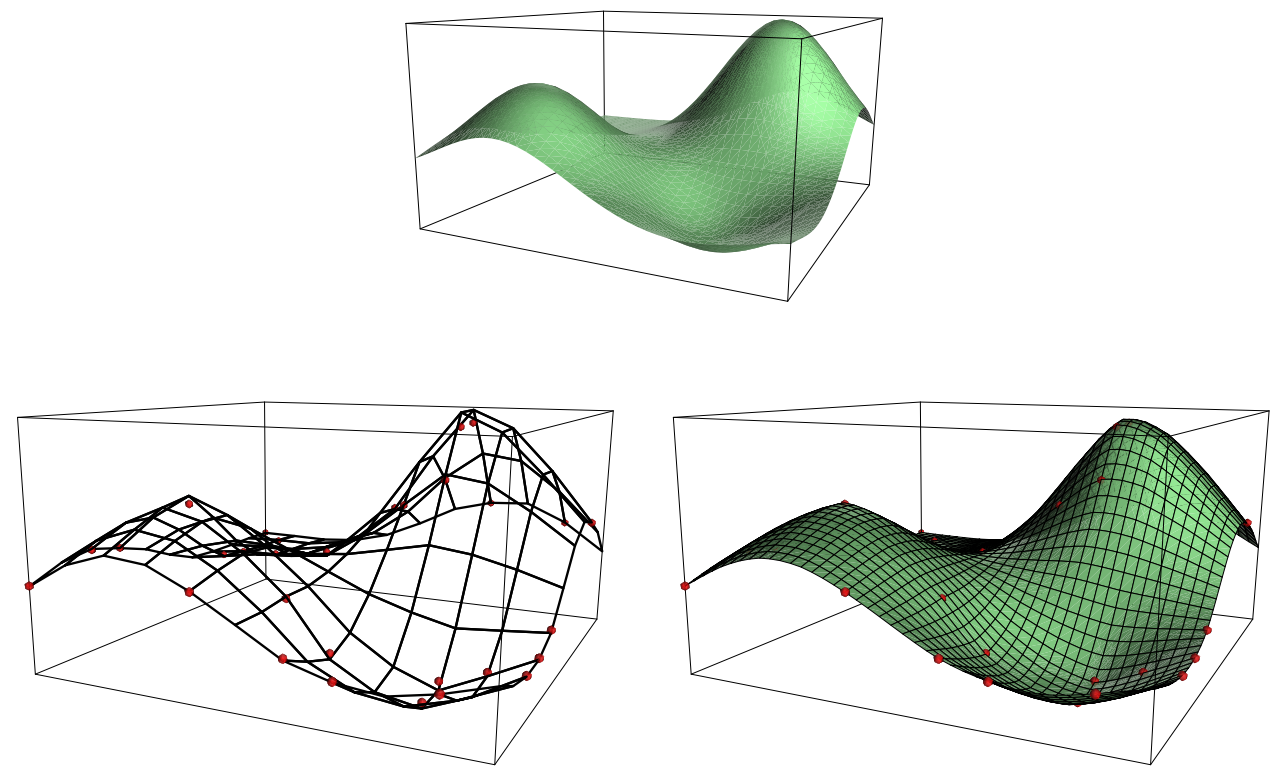

Weimer and Warren, Figure 9: Scattered data interpolation using thin plate spline subdivision. The original surface is shown on the top. Thirty random samples were used to construct the control mesh on the bottom left which results in the interpolating subdivision surface on the bottom right.
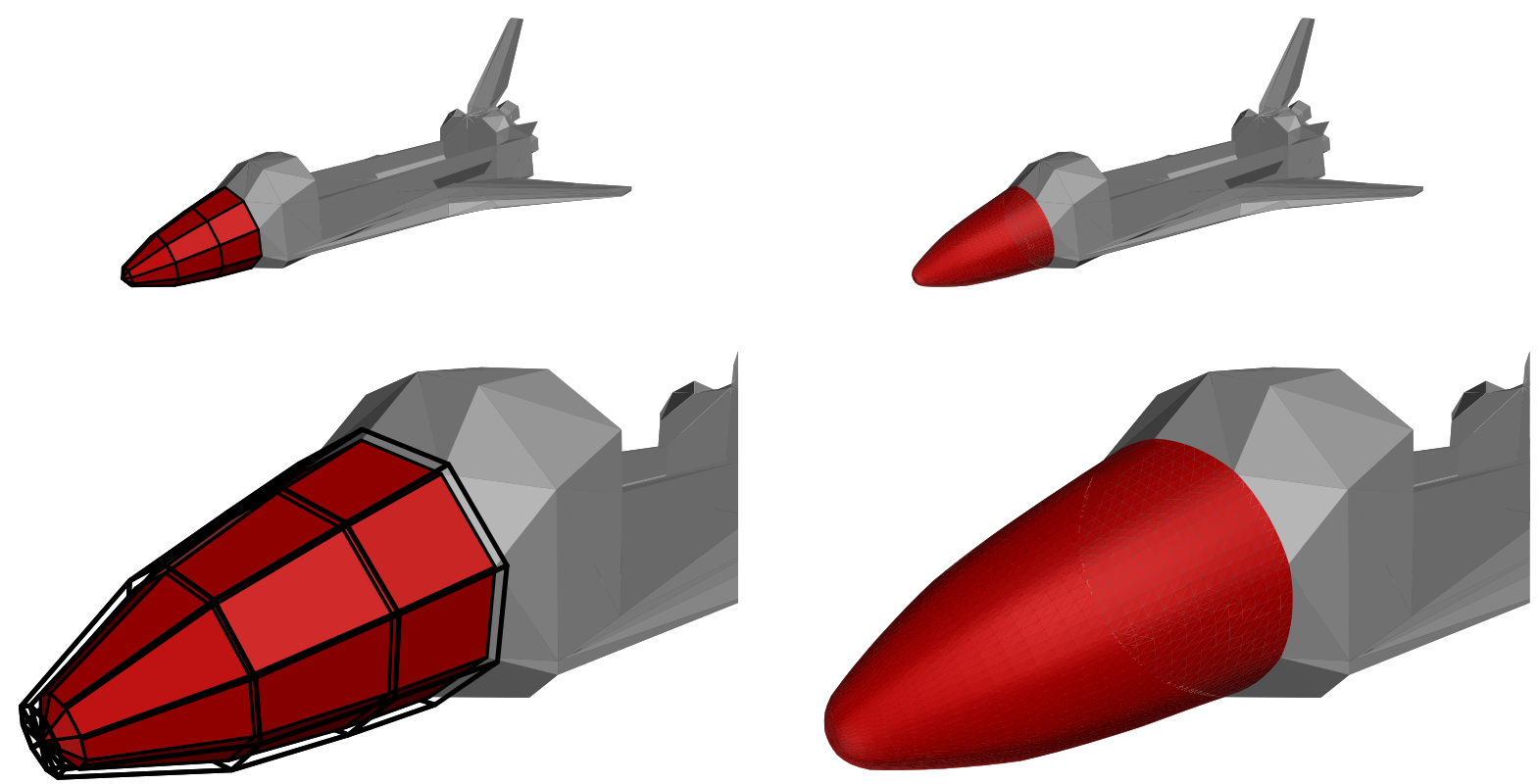

Weimer and Warren, Figure 10: The nose of the original space shuttle model (left) replaced by a fair subdivision surface which interpolates the original vertices (right). The bottom left figure also shows the control polygon produced by the inverse of the interpolation matrix. 